\title{
Conservative Treatment versus Elective Repair of Umbilical Hernia in Patients with Liver Cirrhosis and Ascites
}

\author{
Ashraf Abdel El-hamid Abd El-monem, Mohammed Abbas, Mahmoud Ramadan Abdelaziz \\ El-halawany* \\ Department of General Surgery, Faculty of Medicine, Al-Azhar University \\ *Corresponding author: Mahmoud R.A. El-halawany, E-Mail: dr7alawany@gmail.com
}

\begin{abstract}
Background: management of umbilical hernia in patients with liver cirrhosis remains controversial. The general surgical opinion is that umbilical hernia in patients with liver cirrhosis and ascites has high operative risks and high recurrence rates after elective repair. Conservative treatment, however, can also have severe complications resulting in emergency repair. In these patients such operations carry an even greater risk of complications than compared to elective operations.

Objective: Aiming at comparing between conservative treatment and elective repair of umbilical hernia in patients with liver cirrhosis and ascites.

Patients and Methods: a randomized prospective interventional study Our series was carried out on 40 consecutive patients at General Surgery department, Al-Azhar University hospital; Bab Elshaeria, Cairo, Egypt in the period from June 2017 till September 2018. The patients were divided into 20 patients (group A) undergo surgical repair and 20 patients (group B) undergo conservative treatment. Mean follow up was 6 months.

Results: elective repair of umbilical hernia in patients with liver cirrhosis and ascites had fewer complications than conservative treatment.

Conclusion: Our study showed that elective repair of umbilical hernia in cirrhotic and ascetic patients had less complication than conservative treatment.

Keywords: Conservative Treatment, Elective Repair, Umbilical Hernia, Liver Cirrhosis, Ascites.
\end{abstract}

\section{INTRODUCTION}

An umbilical hernia (UH) occurs through the umbilical fibro-muscular ring, which is usually obliterated by age 2 years. They are congenital in origin and are repaired if they persist in children older than 2-4 years ${ }^{(1)}$. It presents as a central, mid-abdominal bulge. Altered sensorium and obesity enhance incarceration. Hypertrophic, hyperpigmented, papyraceous skin testifies to high pressure being placed on the skin. The size of the fascial defect and whether it is circular provide management clues ${ }^{(2)}$.

Cirrhosis of the liver is a consequence of chronic hepatic injury, with healing by regeneration and fibrosis. Fibrosis leads to further cell damage and destruction of hepatic architecture, progressing to liver failure and portal hypertension ${ }^{(3)}$.

Umbilical hernia is the most frequent abdominal wall complication of ascites in cirrhotic patients ${ }^{(4)}$. Umbilical hernias are common among patients with cirrhosis and uncontrolled ascites. Complications of these hernias can be serious, and mortality rates as high as $30 \%$ have been reported. Skin ulceration and subsequent rupture of the hernia sac and leakage is common, which can result in bacterial peritonitis and serious morbidity ${ }^{(5,6)}$.

The indications for umbilical herniorrhaphy in cirrhotic patients unfortunately remain controversial, as there are no high-quality prospective studies to address this question. Because of this, hernia repair in the presence of advanced cirrhosis and ascites traditionally resulted in high rates of morbidity and mortality, prompting many to avoid elective repair and to operate only when complications develop ${ }^{(5)}$.

Non-operative management of ruptured hernias with antibiotics and dressing changes may result in mortality rates in the range 60$88 \%$. Thus, complicated umbilical hernias in cirrhotics should be repaired urgently ${ }^{(7)}$. Ammar ${ }^{(7)}$ identified wound infections in $6.8 \%$ of their open repairs, while Cassie et al. ${ }^{(8)}$ reported a wound complication rate of $23.3 \%$, with half of those being superficial dehiscence and the other half having wound infections ${ }^{(8)}$. 


\section{AIM OF THE WORK}

The purpose of this study is to compare between conservative treatment and elective repair of umbilical hernia in patients with liver cirrhosis and ascites.

\section{PATIENTS AND METHODS}

Study design: This prospective randomized study was carried out on 40 consecutive patients at General Surgery department Al-Azhar University hospital; Bab Elshaeria, Cairo, Egypt.

Patients were divided randomly into two groups; group A: 20 patients undergo surgical repair, while group B: 20 patients undergo conservative treatment. The study was approved by the Ethics Board of Al-Azhar University.

\section{Patients' selection:}

Inclusion criteria: 1. Primary umbilical hernia. 2. Liver cirrhosis. 3. Ascites (proven or treated). 4. Age $\geq 18$ years. 5. An informed consent was obtained from each patient.

Exclusion criteria: 1. Recurrent umbilical hernia. 2. Midline laparotomy in the medical history. 3. Incarcerated umbilical hernia necessitating an emergency procedure. 4. Expected time to liver transplantation more than 3 months.

\section{RESULTS}

\section{Age and sex:}

The age of patients ranged between 32 and 57 years with a mean age of $44.5 \pm 12.5$ years. Twenty-seven cases were males $(27 / 40,67.5 \%)$ while thirteen cases were females $(13 / 40,32.5 \%)$ with a male to female ratio of $2.15: 1$ and the statistical analysis revealed that males were more commonly affected than females. (Table 1 and Figure 1).
Table 1: Demographic data of the study

\begin{tabular}{|ll|l|}
\hline \multicolumn{2}{|l|}{ Variable } & Value \\
\hline Age & & $44.5 \pm 12.5$ \\
\hline Sex & & \\
& Male & $(27 / 40,67.5 \%)$ \\
& Female & $(13 / 40,32.5 \%)$ \\
\hline
\end{tabular}

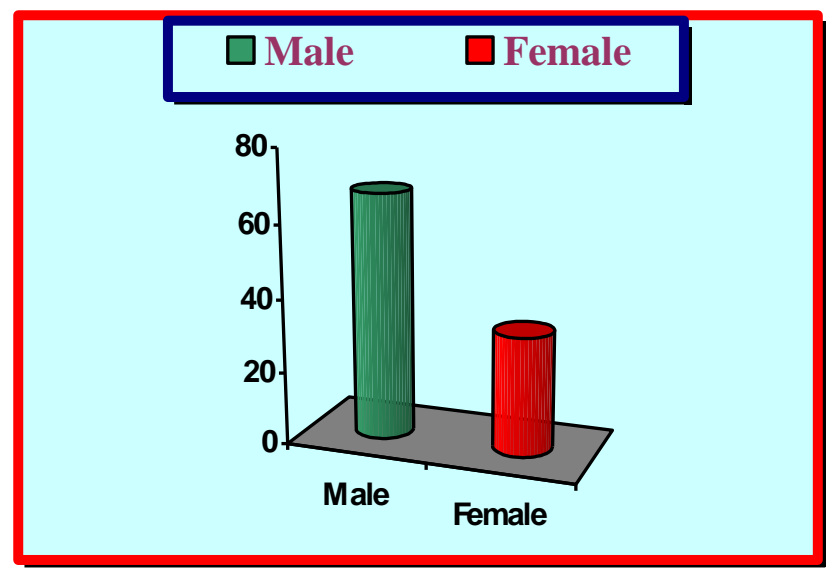

Fig. 1: Sex distribution in patients of the study

The patients was divided into two groups each group 20 patients.

1- Group A was subdivided into two groups (figure 1):

- Group AI was treated surgically without mesh 15 patients (10 males and 5 females).

- Group AII was treated surgically with mesh repair (5 females).

2- Group B: was treated conservatively without surgical intervention (17 males and 3 females). 


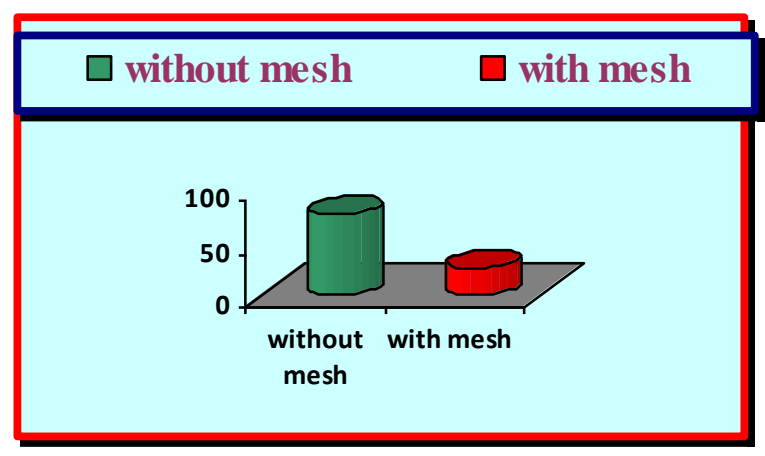

Figure 2: subdivision of group A

Post-operative complications and follow-up:

Early follow up:

During the early post-operative period we had five of our patients $(5 / 20,25 \%)$ had complications; three cases had wound infection $(3 / 20,15 \%)$ that responded well to local wound care and parenteral antibiotics, one patient had ascitic fluid leak from the wound $(1 / 20,5 \%)$ and one patient developed postoperative hematoma $(1 / 20,5 \%)$ that responded well to cold compresses (Table 2 and Fig. 3).

\begin{tabular}{|c|c|c|}
\hline Variable & & Value \\
\hline \multicolumn{3}{|l|}{ Early follow-up: } \\
\hline No complications & & $15(75 \%)$ \\
\hline Complications: & & $5(25 \%)$ \\
\hline & Wound infection & ○ $3(15 \%)$ \\
\hline & Hematoma & $\circ 1(5 \%)$ \\
\hline & Ascitic fluid leak & $\circ 1(5 \%)$ \\
\hline
\end{tabular}

Table 2: Rate of early post-operative complications in patients of the study

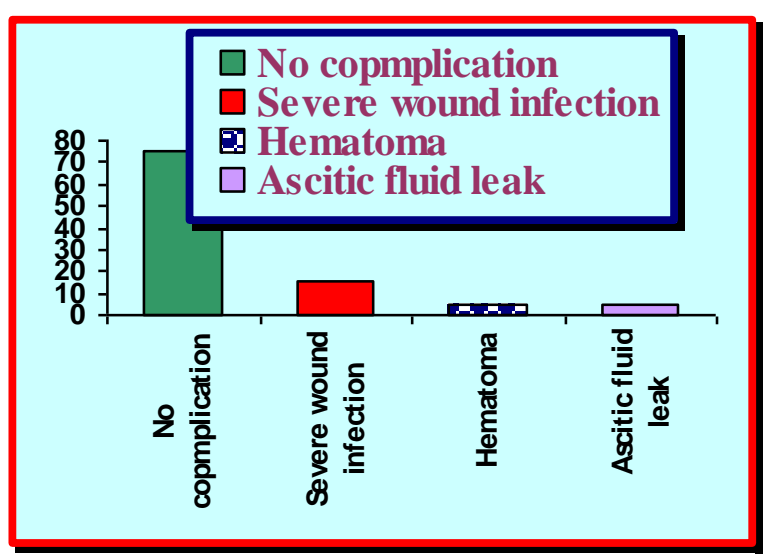

Fig. 3: Rate of early post-operative complications in patients of the study

\section{Late follow up:}

During the follow-up period of 6 months, we had two cases of recurrence $(2 / 20,10 \%)$ due to wound dehiscence and massive ascites development. While there was one patient died $(1 / 20,5 \%)$ due to non-surgical causes; due to hepatic coma (Table 3 and Fig.4).

\begin{tabular}{|c|c|}
\hline Variable & Value \\
\hline $\begin{array}{ll}\text { - } & \text { No complications } \\
\text { - } & \text { Complications } \\
& \circ \quad \text { Recurrence } \\
\text { - } & \text { Died: Hepatic coma }\end{array}$ & $\begin{array}{l}17 / 20(85 \%) \\
2 / 20(10 \%) \\
1 / 20(5 \%)\end{array}$ \\
\hline
\end{tabular}

Table 3: Rate of late post-operative complications in patients of the study

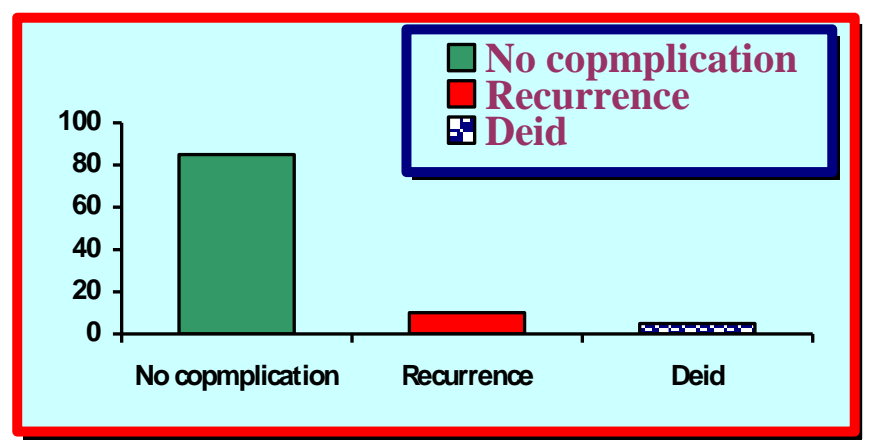

Fig. 4: Rate of late post-operative complications in patients of the study Complication of group B (Conservative group)

During the 6 months, there was significant increase of complications in conservative group when compared to operative group.

We had five patients of group B $(5 / 20,25 \%)$ had incarceration and need emergent surgical intervention, Three cases $(3 / 20,15 \%)$ had infection that respond to parenteral antibiotics, Two cases $(2 / 20,10 \%)$ had ascetic leakage that responded to conservative treatment of ascites and frequent sterile dressing, One case develop hepatic encephalopathy and admitted to ICU (Fig.5). 


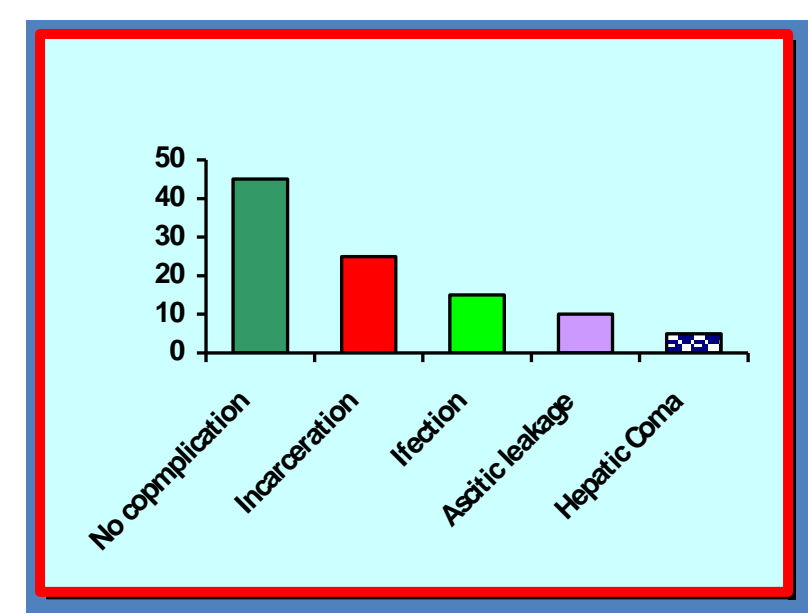

Fig. 5: Rate of complications in patients of group B

\section{DISCUSSION}

Management of umbilical hernia in patients with liver cirrhosis is a subject of debate. Historically, elective hernia repair was deemed hazardous for patients with an umbilical hernia, because of the presumed increased surgical risks and the high recurrence rates after repair. Instead, watchful waiting was often advised, particularly in patients with asymptomatic umbilical hernia ${ }^{(9)}$.

In our study, the age of patients ranged between 32 and 57 years with a mean age of $44.5 \pm 12.5$ years. There was a male predominance as $(27 / 40,67.5 \%)$. Wang and his assistants (10) studied 157 patients with umbilical hernia with cirrhosis and found that a male predominance $63.1 \%$ which was in agreement with our results ${ }^{(10)}$. On the other hand, Andraus and his colleagues ${ }^{(11)}$ showed that the mean age of their patients ranged between 45-60 years with a mean age of 51 years this was not in agreement with our study may be due to endemic disease in Egypt that decrease mean age ${ }^{\mathbf{( 1 1 )}}$.

During the early post-operative period we had five of our patients $(5 / 20,25 \%)$ had complications. Farooqe Dar and his colleagues, in their study found that the early postoperative complications were $(16.1 \%)$ and that was not an agreement with our results ${ }^{(12)}$.

Postoperative wound infection diagnosed by erythema and induration were recorded in three cases $(15 \%)$ without obvious suppuration, all responded well to local wound care and parenteral antibiotics. It appeared that- from our study- there was no statistical significant difference for superficial skin infection to occur in the presence or absence of surgical drain as the hepatic patients had many other risk factors for developing skin infection as: hypoalbumenaemia, malnutrition, hypovitaminosis and hyperglycaemia. Farooqe Dar and his colleagues, in their study found that $(2.9 \%)$ had wound infection ${ }^{(\mathbf{1 2})}$. Hassan and his colleagues, in their study, cases of umbilical hernias with liver cirrhosis found wound infection was (3.9\%) ${ }^{\mathbf{( 1 3})}$.

During the follow up, two cases (10\%) reported to have a recurrent hernia after 6 months postoperative mainly due to partial wound dehiscence and massive uncontrolled ascites development. Otherwise one patient died from causes not related to the surgical technique due to hepatic coma.

Yu and his colleagues, in their study found $(22 \%)$ recurrence rate which is much more than the recurrence rate in our study. They denoted that uncontrollable ascites was responsible for the recurrences ${ }^{(\bullet)}$. McKay and his colleagues documented in their study, that the incidence of recurrence in umbilical hernia with cirrhosis was $(8.51 \%)$ which was in agreement with our results ${ }^{(5)}$.

It seems that the recurrence rate differs according to the number of the patients from one study to another. Multiple risk factors exist for the development of recurrent hernia as patientspecific risks include; advanced age, malnutrition, the amount of ascites, corticosteroid use, cigarette smoking and obesity. Postoperative risks like wound infection is believed to be one of the most significant prognostic risk factors for development of recurrent hernia and technical aspects of wound closure likely contribute to recurrent hernia formation. Wound closed under excessive tension are prone to fascial closure disturbance $^{(\mathbf{1 4})}$.

We had five patients of group B (5/20, 25\%) had incarceration and need emergent surgical intervention, Three cases $(3 / 20,15 \%)$ had infection that respond to parenteral antibiotics, Two cases $(2 / 20,10 \%)$ had ascetic leakage that responded to conservative treatment of ascites and frequent sterile dressing, One case develop hepatic encephalopathy and admitted to ICU. 
Marsman and his colleagues stated that Conservative management of umbilical hernias in patients with liver cirrhosis and ascites leads to a high rate of incarcerations with subsequent hernia repair in an emergency setting, whereas elective repair can be performed with less morbidity and is therefore advocated. That is agrees with our study ${ }^{(15)}$.

\section{CONCLUSION}

Elective repair of umbilical hernia in cirrhotic and ascetic patients unfortunately remain controversial. Our study showed that elective repair of umbilical hernia in cirrhotic and ascetic patients had less complication than conservative treatment.

\section{REFERENCES}

1. Liang MK, Berger RL, Li LT et al. (2013): Outcomes of laparoscopic vs open repair of primary ventral hernias. JAMA Surg., 148: 1043-1080.

2. Fitch MT and Manthey DE (2013): Abdominal Hernia Reduction. In: "Roberts. Roberts and Hedges' Clinical Procedures in Emergency Medicine", 6th ed., New York: Saunders, pp 873-879.

3. Nusrat S, Khan MS, Fazili J et al. (2014): Cirrhosis and its complications: evidence based treatment. World J. Gastroenterol., 20: 5442-5460.

4. Dokmak S, Aussilhou B, Belghiti J (2012): Umbilical hernias and cirrhosis. J. Visc Surg., 149(5): 32-39.

5. McKay A, Dixon E, Bathe $O$ et al. (2009): Umbilical hernia repair in the presence of cirrhosis and ascites: results of a survey and review of the literature. Hernia, 13: 461-468.

6. Yu BC, Chung $M$ and Lee $G$ (2015): The repair of umbilical hernia in cirrhotic patients: 18 consecutive case series in a single institute. Ann Surg Treat Res., 89: 87-91.
7. Ammar SA (2010): Management of complicated umbilical hernias in cirrhotic patients using permanent mesh: randomized clinical trial. Hernia, 14: 3538.

8. Cassie S, Okrainec A, Saleh F et al. (2014): Laparoscopic versus open elective repair of primary umbilical hernias: short-term outcomes from the American College of Surgeons National Surgery Quality Improvement Program. Surg Endosc., 28: 741-746.

9. Leonetti JP, Aranha GV, Wilkinson WA et al. (1984): Umbilical herniorrhaphy in cirrhotic patients. Arch Surg., 119(4): 442-445.

10. Wang R, Qi X, Peng Y et al. (2016): Association of umbilical hernia with volume of ascites in liver cirrhosis: a retrospective observational study. J Evid Based Med., 9: 170-180.

11. Andraus W, Soares R, Pinheiro1 Q (2015): Abdominal wall hernia in cirrhotic patients: emergency surgery results in higher morbidity and mortality. BMC Surgery, 15: 65-71.

12. Farooq Dar M, Butt MQ, Sheen SN et al. (2016): Hernia repair in patients with chronic liver disease. Pak. Armed Forces Med J., 66: 395-399.

13. Hassan AA, Salama AF, Hamdy H et al. (2014): Outcome of sublay mesh repair in non-complicated umbilical hernia with liver cirrhosis and ascites. Intl J Surg., 12: 181-185.

14. Mansour A, Watson W, Shayani $\mathrm{V}$ et al. (1997): Abdominal operations in patients with cirrhosis: still a major surgical challenge. Surgery, 122:730-735.

15. Marsman HA, Heisterkamp J, Halm JA et al. (2007). Management in patients with liver cirrhosis and an umbilical hernia. Surgery, 142(3): 372-5. 